\title{
Anomalous diffusion and ballistic peaks: A quantum perspective
}

\author{
M. Stefancich, ${ }^{1}$ P. Allegrini, ${ }^{1}$ L. Bonci, ${ }^{1}$ P. Grigolini,,${ }^{1,2,3}$ and B. J. West ${ }^{1}$ \\ ${ }^{1}$ Center for Nonlinear Science, University of North Texas, P.O. Box 5368, Denton, Texas 76203 \\ ${ }^{2}$ Dipartimento di Fisica dell'Università di Pisa, Piazza, Torricelli 2, 56127 Pisa, Italy \\ ${ }^{3}$ Istituto di Biofisica del Consiglio Nazionale delle Ricerche, Via San Lorenzo 26, 56127 Pisa, Italy
}

(Received 15 December 1997)

\begin{abstract}
The quantum kicked rotor and the classical kicked rotor are both shown to have truncated Lévy distributions in momentum space, when the classical phase space has accelerator modes embedded in a chaotic sea. The survival probability for classical particles at the interface of an accelerator mode and the chaotic sea has an inverse power-law structure, whereas that for quantum particles has a periodically modulated inverse power law, with the period of oscillation being dependent on Planck's constant. These logarithmic oscillations are a renormalization group property that disappears as $\hbar \rightarrow 0$ in agreement with the correspondence principle.
\end{abstract} [S1063-651X(98)14406-9]

PACS number(s): 05.45.+b, 03.65.Sq

\section{INTRODUCTION}

In the past two decades classical mechanics has emerged as an area of fundamental study in three distinct domains. The most familiar is that of regular, predictable motion, the so-called integrable Hamiltonian systems with KolmogorovArnold-Moser (KAM) tori on the energy shell. The most exotic are the completely nonintegrable Hamiltonian systems in which trajectories exponentially separate from one another. If a dynamical system such as a standard map is fully chaotic, meaning that all the KAM tori have become globally unstable and disintegrated, producing a chaotic sea in phase space then the mean-square momentum of the system increases linearly in time. Such classical systems are said to be diffusive and this relation between statistics and dynamics has been understood for nearly two decades [1]. The third and largest category of motion is called weakly chaotic and contains aspects of both regular and chaotic motion in that there are islands of KAM tori in a sea of chaos. The dynamical orbits can, rather than exponentially separating as they do in the case of strong chaos, stick to the cantori at the phase space boundary between stable islands and the chaotic sea in weakly chaotic systems [2] with a resulting anomalous diffusion, i.e., diffusion that can be either faster or slower than normal.

Here we investigate the connection between a nonintegrable classical Hamiltonian system, in the weak chaos case, and its corresponding quantum system. We wish to understand the sense in which a classical chaotic solution is the limit of the corresponding quantum solution to the Schrödinger equation as $\hbar \rightarrow 0$. The resolution of this question bears on how good any semiclassical approximation is to the solution of quantum problems. For the sake of generality we study the paradigm of how chaos arises in simple Hamiltonian systems, namely, the standard map [1-3]:

$$
\begin{gathered}
p_{n+1}=p_{n}-K \sin \theta_{n}, \\
\theta_{n+1}=\theta_{n}+p_{n+1},
\end{gathered}
$$

where $p_{n}$ is the scaled angular momentum after the $n$th kick and $\theta_{n}$ is the corresponding angle with period $2 \pi$. Equation (1) describes the classical kicked rotor (CKR) as discussed in Sec. III, and is also the basis of the quantum kicked rotor (QKR) using the Schrödinger equation as discussed in Sec. IV. The numerical calculations of the CKR and QKR are presented in these sections, respectively, and their results compared in Sec. V.

The heart of our analysis is the numerical investigation of the properties of ensembles of trajectories in the phase space where accelerator modes are present, such as depicted in Fig. 1. Unlike the strong chaos case where the momentum grows diffusively, in the situation depicted in Fig. 1 the growth in momentum is anomalous. The problem of anomalous diffusion in the kicked rotor occurs for a small region of parameter values that otherwise correspond to fully developed chaos. In this region the mean-square momentum increases in time as $t^{2 H}$ with $H \neq \frac{1}{2}$ but like normal diffusion this anomaly is eventually quenched by quantum effects [4]. The transition from normal diffusion to localization [5,6] and the transition from anomalous to normal diffusion [4] has been

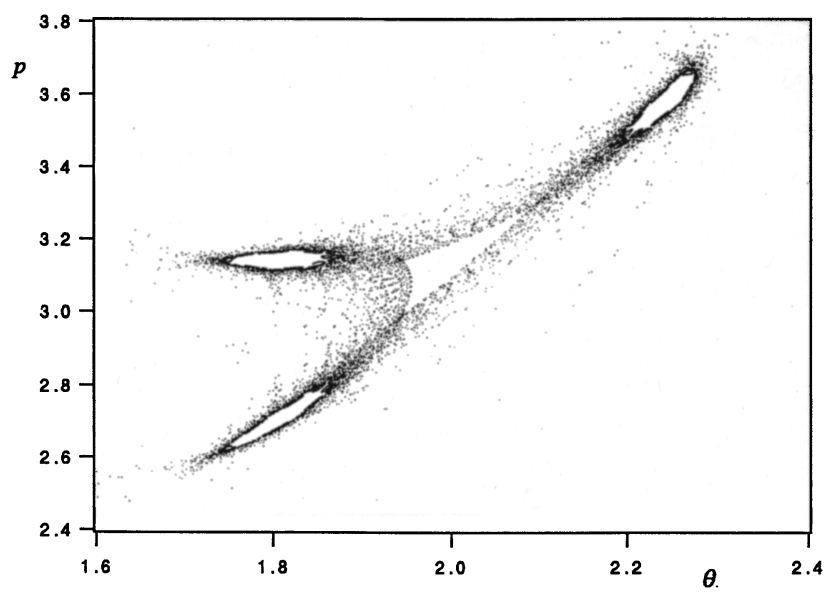

FIG. 1. The phase space representation of an accelerator mode for the CKR. The values of the nonlinear parameter in this and in the following figures is $K=6.9115$. $\theta$ and $p$ are the dimensionless quantities appearing in Eq. (1). 
analyzed in the QKR to gain insight into the validity of the semiclassical approximation in chaotic systems [3-6]. The exponent $H$ can be related to the fractal dimension of the underlying process and its statistics are often described using nondiffusive Lévy stable processes [7-9]. This has led to studies showing that certain deterministic, nonlinear, dynamical equations with chaotic solutions have statistics described by Lévy stable distributions [10] and scaling that obeys renormalization group relations [11].

Hanson, Ott, and Antonsen [3] investigate, using numerical calculations, the effect of finite $\hbar$ on the manner in which tiny integrable regions of phase space influence the statistical description of the evolution of the QKR. The integrable regions are small, relatively stable KAM islands in phase space for the CKR that are referred to as accelerator modes, since they are in the neighborhood of accelerating fixed points [2], cf. Fig. 1. Such fixed points and the corresponding modes cause the trajectories falling in their neighborhood to be trapped for long times and accelerated (so that their momentum increases linearly in time) and consequently the global mean-square momentum increases faster than linearly. In the classical case the number of particles in an accelerator mode is known to decay as an inverse power law in time [2]. In the quantum case it was found that for large values of $\hbar$ in the numerical calculations, the survival probability density decreases exponentially in time with a rate of decay proportional to $\exp [-1 / \hbar]$. This dependence of the rate on the value of Planck's constant led Hanson, Ott, and Antonsen [3] to interpret the exponential decrease in time of the survival probability as a tunneling effect. On the other hand, by decreasing $\hbar$ a transition value was found after which the decay of the probability was no longer exponential. This transition has an interpretation in terms of time scales such that the exponential decay occurs for early times, those times associated with large values of $\hbar$, and this other decay occurs for later times associated with smaller values of $\hbar$. We are herein concerned with this different behavior and find this decay to be inverse power law. The scaling, as manifest in the inverse power law, suggests a renormalization group description for quantum phenomena.

To study how anomalous diffusion or, equivalently, the Lévy distribution, arises in quantum dynamical systems we present equivalent calculations for the CKR and the QKR and compare the corresponding survival probabilities as $\hbar$ is varied. It is well known that with the choice of strength parameter, $K=6.9115$ in Eq. (1), that gives rise to accelerator modes, the CKR generates highly correlated fluctuations in the momentum variable leading to anomalous diffusion with $H=1-\beta / 2$. Here $\beta$ is the index of the inverse powerlaw memory function that can be associated directly with the slope of the inverse power-law decay of the particle population trapped in the accelerating modes $[2,10,12]$. Only those trajectories trapped by the accelerator modes contribute to the anomalous diffusion and there are always two such modes, one accelerating particles to positive momentum and the other accelerating particles to negative momentum. Therefore, disregarding the irrelevant trajectories that are moving erratically in the chaotic sea, we consider this dynamical model, the CKR, to be a generator of a virtually dichotomous fluctuation process. Thus, we examine a twostate stochastic model in Sec. II to further our understanding of both the CKR and QKR. In fact we find that all three have the same basic dynamical evolution, that being a truncated Lévy distribution with a propagating peak at both its positive and negative fronts.

In Sec. V we determine that both the CKR and the QKR have a survival probability that decays as an inverse power law for $\hbar \rightarrow 0$. However, in the quantum case we find that the inverse power law has a modulation that is periodic in the logarithm of time with a period of oscillation that diverges as $\hbar^{-\alpha}$ where $\alpha \approx 0.14$. This dependence is described by a postulated quantum coherency mechanism that retains the scaling property of the cantori in the vicinity of the accelerator mode, while yielding a survival probability that goes over to the appropriate classical result as $\hbar \rightarrow 0$. In Sec. VI we formulate some conclusions based on these calculations regarding the correspondence principle and microscopic scaling.

\section{GOLD MODEL}

The statistical approach to classical anomalous diffusion has a long history and has recently been the subject of a number of papers; see, for example, Refs. [12-14]. This stochastic perspective rests on the simple one-dimensional differential equation:

$$
\frac{d x}{d t}=\xi(t)
$$

Of special interest is the case where $\xi(t)$ is a two-state stochastic process taking the values $\pm W$. The evolution of the probability density $p(x, t)$, where $p(x, t) d x$ is the probability that the dynamical variable is in the interval $(x, x+d x)$ at time $t$, is given by the exact integrodifferential equation $[12,14]$,

$$
\frac{\partial}{\partial t} p(x, t)=\int_{0}^{t}\left\langle\xi(t) \xi\left(t^{\prime}\right)\right\rangle \frac{\partial^{2}}{\partial x^{2}} p\left(x, t^{\prime}\right) d t^{\prime} .
$$

It is clear from Eq. (3) that the character of the diffusion depends on the choice of the correlation for the $\xi$ fluctuations in Eq. (2). Normal diffusion is a consequence of the existence of a microscopic time scale, defined by

$$
\tau=\int_{0}^{\infty} \Phi_{\xi}(t) d t
$$

where $\Phi_{\xi}(t)$ is the stationary two-time $\xi$-correlation function. If the correlation decays quickly enough to make $\tau$ finite, we can explore the random-walk process for times $t$ very large compared to $\tau$. The time scale separation between the random-walk process and the velocity fluctuations allows the central limit theorem to work, so that for $\tau<\infty$ a Gaussian diffusion process for the two-state model is eventually reached. The diffusion is anomalous if the correlations of the fluctuations are stationary and have the asymptotic inverse power-law form

$$
\Phi_{\xi}(t)=\frac{\langle\xi(t) \xi(0)\rangle}{\left\langle\xi^{2}\right\rangle}=\frac{A}{\left(A^{1 / \beta}+t\right)^{\beta}}
$$

and the power-law index is in the range 


$$
0<\beta<1 \text {. }
$$

When $\tau \rightarrow \infty$, as Eq. (3) does for the inverse power law with an index in the range (6), there is no time scale separation between the macroscopic (diffusive) and the microscopic processes (fluctuations of $\xi$ ) and the resulting statistics are not Gaussian in general. In fact it has been shown that under certain conditions, that is, when the constant in the denominator in Eq. (5) can be neglected, Eq. (3) is a fractional derivative whose solution is bimodal [14].

The model given by Eq. (3) with condition (5) has so far been discussed in a stochastic or random-walk context. It has been shown elsewhere that there is also a dynamical realization of this model [12]; one in which the stochastic nature of the velocity is generated by an intermittent chaotic map, namely, the map of Geisel, Nierwetberg, and Zacherl [15]. This relation was established by Zumofen and Klafter [16] where they showed that such a map produced a long-time $\alpha$-stable Lévy process. Such a Lévy process, defined as the solution of the Bachelier-Smoluchowski-ChapmanKolmogorov (BSCK) chain condition, is Markovian [7], but the variable $x$, at any finite time, can change by an arbitrarily large amount, with a given inverse power-law distribution. The process described by Eq. (3), supplemented by Eqs. (5) and (6), is by contrast non-Markovian. However, it is possible to prove [12] that the statistical equivalence of this non-Markovian process with an $\alpha$-stable Lévy process is guaranteed by the possibility of replacing the time nonlocality of the dynamical process with a space nonlocality. This interchange of space and time produces the same effect as a process that is originally nonlocal in space. Yet, at every finite time, the equivalence of the solution to Eq. (3) with the "true", Lévy process cannot be complete, because a Lévy process is characterized by infinite central moments of $x$; whereas in the random-walk approach (2), with excursions based on steps having a finite speed $W$, the distribution of the diffusing variable at time $t$ is truncated at $|x|=W t$. No trajectory can travel, in a time $t$, a distance larger than $|x|$ $=W t$, corresponding to the rare event in which $\xi(t)$ does not change sign for the entire time interval, implying, at every finite time, an accumulation of particles at $x=-W t$ and at $x=+W t$. This accumulation results in two intense peaks for the distribution of the diffusing variable $x$, as clearly shown by the results of numerical calculations $[12,17]$, see also Fig. 2. Nonetheless, the distribution will asymptotically become an $\alpha$-stable Lévy process as the peaks recede to infinity and their amplitudes fade to zero. Since this model has a truncated Lévy distribution as a solution it has been called the generator of Lévy distributions (GOLD) model [18].

The one-dimensional, centrosymmetric form of the Lévy distribution is given by

$$
p_{\mu}(x, t)=\frac{1}{2} \int_{-\infty}^{\infty} e^{i k x} e^{-b t|k|^{\mu}} d k,
$$

where $b>0$. Only a handful of explicit forms of the Lévy distribution are known for specific values of the Lévy index, for example, the Gaussian for $\mu=2$ and the Cauchy for $\mu=1$. In terms of Eq. (7), where $1 \leqslant \mu \leqslant 2$ in the present case [12], we can write for the truncated Lévy distribution

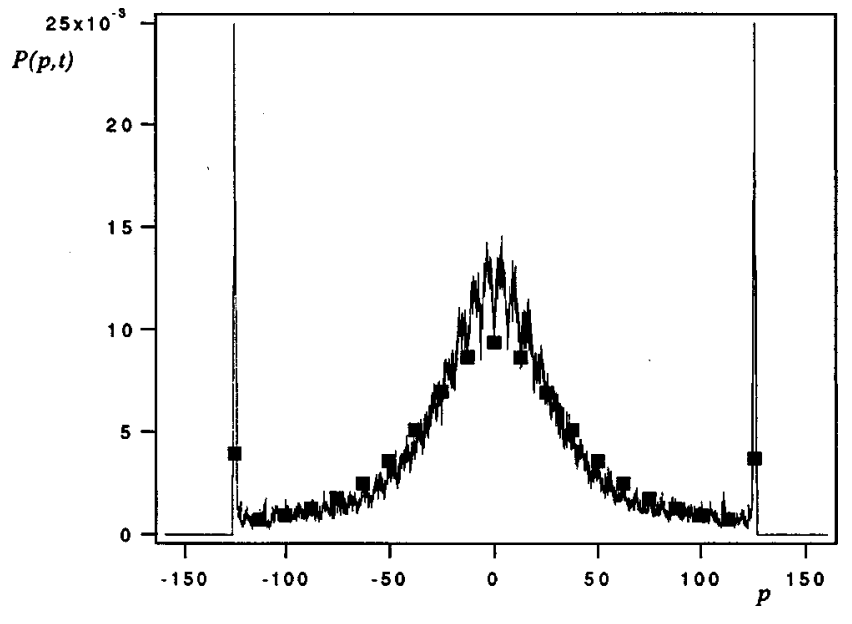

FIG. 2. Classical distribution $P(p, t)$ after 20 iterations of the map (1), continuous line, and the theoretical prediction of Eq. (8), boxes. The quantity $p$ is the dimensionless momentum from Eq. (1) and the probability distribution is normalized to 1 .

$$
\begin{aligned}
p(x, t)= & p_{\mu}(x, t) \Theta(|x|-W t)+\frac{\Pi(t)}{2}\{\delta(x+W t) \\
& +\delta(x-W t)\}
\end{aligned}
$$

where $\Theta(\cdot)$ is the Heaviside unit step function; the amplitude of the peaks is determined by the correlation function (5),

$$
\Pi(t)=k \Phi_{\xi}(t),
$$

and $k$ is a constant that is determined by the specific realization of the numerical calculation. There is excellent agreement between the form of the solution given by Eq. (8) and numerical experiments on the CKR as shown in Fig. 2, see Ref. [18]. Thus, we have that the GOLD model results in a truncated Lévy distribution and is characterized by two ballistic peaks, whose intensity decays in time as the correlation function (5).

\section{NUMERICAL RESULTS FOR THE CKR}

The Hamiltonian for the classical kicked rotor is

$$
H=\frac{P^{2}}{2 I}-I \omega_{0}^{2} \cos \theta \sum_{j=-\infty}^{\infty} \delta\left(j-\frac{t}{T}\right),
$$

where $\theta$ is an angular coordinate of period $2 \pi, P$ is the angular momentum, $I$ is the moment of inertia, $\omega_{0}$ is the natural frequency of small oscillations, and $T$ is the time interval between kicks. The integration of Hamilton's equations of motion over a single period of a kick yields the standard map given by Eq. (1), where the strength of the kick is $K=\omega_{0}^{2} T^{2}$ and the scaled momentum is given by $p$ $=P T / I$. Thus, the evolution is completely determined by the numerical value of the parameter $K$.

We iterate the standard map for a value of the strength parameter, $K=6.9115$, to obtain the accelerator mode indicated in Fig. 1 from the trajectories in the vicinity of the accelerator fixed point $(\pi / 4,0)$. It is apparent from this figure that the KAM islands are encircled by cantori that separate the accelerator mode from the chaotic sea. These phase space 
structures manifest a self-similarity, see, for example $[9,17,19]$, and tend to entrap orbits that approach the accelerator mode too closely.

A careful numerical study of anomalous diffusion in the CKR due to accelerator modes in the standard map was made by Ishizaki and co-workers [19]. These authors established that the exponent characterizing anomalous diffusion $\left\langle p^{2}(t)\right\rangle \propto t^{2 H}$ and the exponent of the correlation function $\Phi_{\xi}(t) \propto 1 / t^{\beta}$ are related by $H=1-\beta / 2$. Further, they found that the waiting time distribution index, $\phi(t) \propto 1 / t^{\mu}$, is found to be $\mu=2+\beta=4-2 H$. The waiting time distribution comes from continuous time random-walk theory and determines the probability that a walker (orbit) will wait a time between $t$ and $t+d t$ in its present state before making a transition to the other state [17]. Ishizaki and co-workers make a careful theoretical and numerical analysis of the momentum distribution to verify these relations among the exponents. A direct numerical evaluation of the waiting time distribution $\phi(t)$ was made by Klafter, Zumofen, and Shlesinger [17]. These latter calculations spanned six decades in time scale, and resulted in slight and seemingly irregular oscillations of the slope around the value of $\mu$ in the theoretical inverse power-law form of $\phi(t)$, but the value was consistent with that found for $H$ in the corresponding study of momentum diffusion [19].

According to the theory of Allegrini, Grigolini, and West [12] sketched in Sec. II, the intensity of the ballistic peaks delimiting the extension of the Lévy distribution was also observed numerically by Zumofen and Klafter [9] using a completely different argument. The relation between the peaks and the correlation function, $\Pi(t)$, depicted in the solution to the GOLD model, Eq. (8), suggests that we need only examine the behavior of the peaks in the CKR calculations to determine their long-time correlation properties. This assumption turns out to be quite good and results in a greatly enhanced efficiency in the calculations, especially for those of the QKR presented in the next section.

The inverse power-law form of the classical waiting time distribution function was first determined by Hanson, Cray, and Meiss [20] in the context of partially destroyed KAM tori. They made use of the self-similar system of rate equations for the fluxes across the zones of quasistability surrounding the accelerator modes and simplified the picture by assuming that successive crossings of these cantori can be treated as a Markov process. The chaotic dynamics thus become statistically equivalent to a random walk on a hierarchial lattice or Markov tree. Here we follow Klafter, Zumofen, and Shlesinger [17] and simplify the argument even further by introducing a hierarchy of time intervals to approximate, by a sum of exponentials, the probability density $\phi(t)$ of a trajectory to enter the system of belts of cantori from the chaotic sea and to stay in the cantori system for a time $t$ before returning to the chaotic sea:

$$
\phi(t)=\sum_{n=1}^{\infty}\left(w^{2} q\right)^{n} \exp \left[-w^{n} t\right],
$$

where $w$ is a rate and $q$ is the probability of that rate occurring in the process of interest. The dominant behavior of the probability density (11) is determined by scaling time with the rate $w$ to obtain the approximate scaling relation $q w^{2} \phi(w t) \approx \phi(t)$.

Consider a function $F(t)$ that is homogeneous so that for two parameters $a$ and $b$ we have

$$
F(b t)=a F(t) .
$$

This equation has a solution of the form [21]

$$
F(t)=\frac{A(t)}{t^{\alpha}},
$$

where by substitution of Eq. (13) into Eq. (12) and equating terms we find that the power-law index is

$$
\alpha=\ln a / \ln b
$$

and the function $A(t)$ is periodic in $\ln t$ with period $\ln b$. Thus, the scaling equation for the probability density (11) has an inverse power-law solution $\phi(t)=A(t) / t^{\mu}$, where the power-law index is given by $\mu=2+\ln q / \ln w$, correcting a typo in Ref. [17]. The probability density $\phi(t)$ is identical to the waiting time distribution, and the second derivative of the waiting time distribution yields the correlation function [12]. On the other hand, the two time integrations necessary to derive $\phi(t)$ from $\Phi_{\xi}(t)$ do not alter the structure of the power-law modulation. To see this take the derivative of Eq. (13)

$$
\frac{d}{d t} \frac{A(t)}{t^{\alpha}}=\frac{d A(t) / d t-\alpha A(t)}{t^{\alpha+1}} \equiv \frac{G(t)}{t^{\alpha+1}},
$$

where $G(t)$ is defined by the numerator of the middle equation, and it is evident that $G(t)$ is periodic in $\ln t$ with period $\ln b$. Thus, the only qualitative difference between the original function $F(t)$ and its derivative is that the index on the inverse power law has increased by 1 . This means that if we choose for the correlation function

$$
\Phi_{\xi}(t)=\frac{g(t)}{t^{\beta}}
$$

the waiting time distribution function would be

$$
\phi(t)=\frac{h(t)}{t^{\beta+2}}
$$

and both $g(t)$ and $h(t)$ maintain the logarithmically periodic structure with the same period. In this way we can relate the index of the inverse power-law correlation function with the parameters in Eq. (11) to obtain $\beta=\ln q / \ln w$. Thus, we can relate the phenomenological description of the waiting time in the CKR to the exact result of the GOLD model.

To verify the prediction that $\beta=\ln q / \ln w$ in the case of the standard map, we used as an initial condition a Gaussian distribution centered near the accelerator mode. This choice mimics a coherent quantum state with $\hbar=0.005$ and is compared with the subsequent quantum calculation. We numerically follow only those orbits that sticks to the accelerator mode, and neglect the other trajectories, that is, we follow the ballistic peaks. The calculated area under the ballistic 


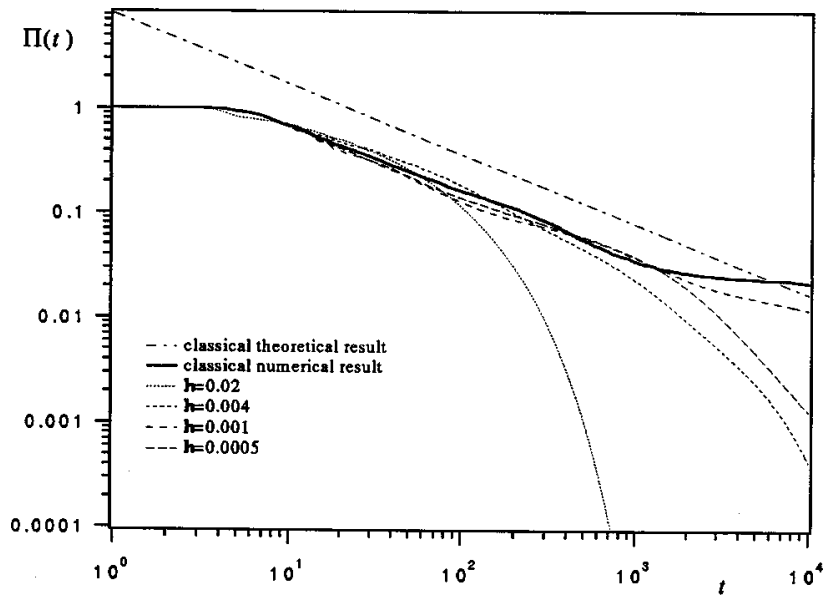

FIG. 3. Population of the peak $\Pi(t)$ as a function of time. The solid line denotes the classical result while the dashed lines are quantum calculations for different values of $\hbar$. The dotted-dashed line indicates the theoretical prediction of [9]. The time $t$ is expressed in the number of kicks and the normalization of $\Pi(t)$ is $\Pi(1)=1$.

peaks as a function of time is depicted in Fig. 3. The slope of the curve is remarkably close to $\beta=-0.67$ the value predicted by the numerical calculation of $H$ via the second moment of the mean square momentum $[17,19]$. The broadened initial condition implies that a given number of trajectories are located within the stable part of the accelerator mode. These trajectories remain confined there forever, and are responsible for a permanent ballistic peak, which is perceived as a long-time plateau depicted in the solid curve. Without such trajectories in the plateau there would be an eventual crossover at long time to an exponential decay. This latter decay is related to round-off errors depending on the computer precision. We note in fact that round-off errors are indistinguishable from externally generated random fluctuations of a given intensity, the effect of which is known to be a crossover from the inverse power-law behavior to an exponential one [22,23]. At the same time, we note that the calculated curve does not imply oscillations as intense as those of Ref. [9], probably as a consequence of the fact that the method of following the peak is more reliable than doing the full calculation at each time step.

\section{NUMERICAL RESULTS IN THE QUANTUM CASE}

We now turn our attention to the QKR. The Hamiltonian is, of course, the same as that in the previous section and we now wish to numerically integrate the Schrödinger equation for the kicked rotor,

$$
\begin{aligned}
i \hbar \frac{\partial}{\partial t} \psi(\theta, t)= & -\frac{\hbar^{2}}{2 I} \frac{\partial^{2}}{\partial \theta^{2}} \psi(\theta, t)-I \omega_{0}^{2} \cos \theta \\
& \times \sum_{j=-\infty}^{\infty} \delta(j-t / T) \psi(\theta, t) .
\end{aligned}
$$

Following Hanson, Ott, and Antonsen [3] we introduce the scaled time $\bar{t}=t / T$ and the scaled period $\tau=\hbar T / I$ so that Eq. (18) reduces to

$$
i \tau \frac{\partial \psi}{\partial \bar{t}}=-\frac{\tau^{2}}{2} \frac{\partial^{2} \psi}{\partial \theta^{2}}-\bar{K} \cos \theta \sum_{j=-\infty}^{\infty} \delta(\bar{t}-j) \psi
$$

Here there are two independent parameters rather than one as in the classical case. The classical parameter $K=\bar{K} \tau$ determines the onset of chaos at $K \approx 0.96$ and as is well known for large values of $K$ the diffusion coefficient is given by $D$ $\approx K^{2} / 2$ [1]. The scaled period $\tau$ is proportional to Planck's constant and is therefore a quantum parameter that vanishes in the classical limit. We denote the expansion of the wave function in the basis of the eigenfunctions of the angular momentum operator $\left\{\psi_{m}\right\}$,

$$
|\psi(t)\rangle=\sum_{m} C_{m}(t)\left|\psi_{m}\right\rangle
$$

The periodicity of the Hamiltonian (10) enables us to describe the quantum evolution in terms of Floquet theory. Within the framework of the Floquet formalism the time evolution is determined via successive application of a onecycle unitary propagator $\hat{C}$, which maps the wave function at time $t$ into the wave function at time $t+T$. The eigenvalue problem for the propagator $\hat{C}$ may be written as

$$
\hat{C}\left|\lambda_{n}\right\rangle=\exp \left[-i E_{n}\right]\left|\lambda_{n}\right\rangle,
$$

where the $E_{n}$ are called quasienergies and for a bounded system are real numbers. The corresponding eigenfunction $\left|\lambda_{n}\right\rangle$ are referred to as quasienergy states or Floquet states. It may be shown that $\left|\lambda_{n}\right\rangle$ are the eigenvectors of the Hermitian operator $\hat{H}-i \hbar \partial / \partial t$ and consequently form a complete orthonormal basis. To obtain the matrix representation of $\hat{C}$ in the angular momentum eigenbasis notice that

$$
\left\langle\psi_{j} \mid \psi(T)\right\rangle=\sum_{k}\left\langle\psi_{j}|\hat{C}| \psi_{k}\right\rangle\left\langle\psi_{k} \mid \psi(0)\right\rangle
$$

and if we chose the initial state such that

$$
\left\langle\psi_{k} \mid \psi(0)\right\rangle=\delta_{k, j^{\prime}},
$$

then

$$
\left\langle\psi_{j} \mid \psi(T)\right\rangle=\left\langle\psi_{j}|\hat{C}| \psi_{j^{\prime}}\right\rangle
$$

Thus the $j^{\prime}$ column of the matrix representation of $\hat{C}$ may be calculated using the initial condition (23) and integrating the time-dependent Schrödinger equation over one period of the perturbation. We can then numerically solve the eigenvalue problem (21). For times $t=k T, k=1,2, \ldots$ the wave function may be written as

$$
|\psi(k T)\rangle=\sum_{m} \exp \left[-i E_{m} k\right]\left|\lambda_{m}\right\rangle\left\langle\lambda_{m} \mid \psi(0)\right\rangle .
$$

It is apparent from Eq. (25) that only the Floquet states overlapping the initial wave function contribute to its subsequent time evolution. These states are frequently referred to as the Floquet spectrum of the wave function. 


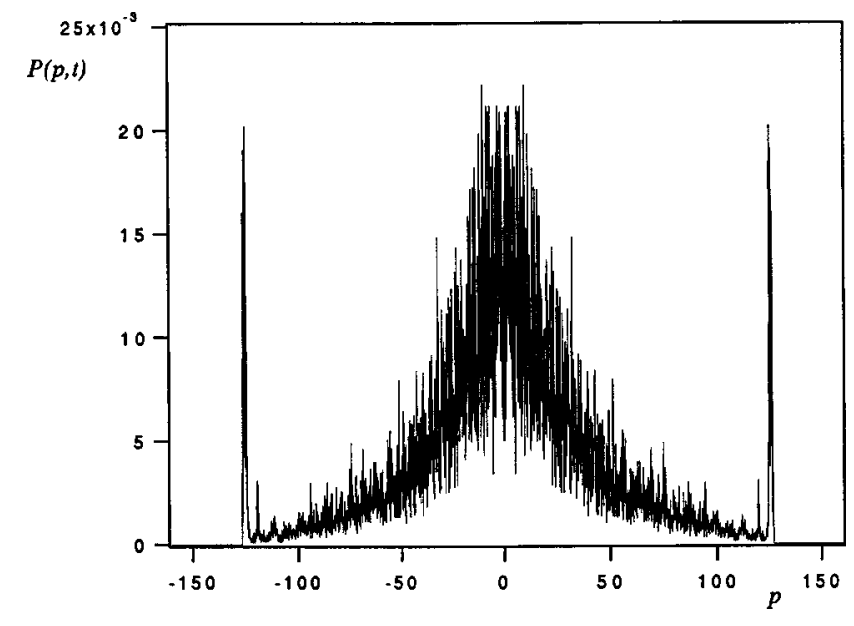

FIG. 4. Quantum probability density after 20 iterations for the QKR, to be compared with its classical counterpart in Fig. 2.

Exploiting the kicked nature of the system we can cast the quantum time evolution as the application of the following mapping to the wave function in the momentum representation:

$$
C_{m}\left(t_{n+1}^{-}\right)=\sum_{m^{\prime}}(i)^{m^{\prime}} J_{m^{\prime}}\left(\frac{K}{\hbar}\right) C_{m+m^{\prime}}\left(t_{n}^{+}\right)
$$

where $J_{m}$ denotes the Bessel function of order $m$ and $\left\{C_{m}(t)\right\}$ are the expansion coefficients in the momentum basis at time $t$, see for example [24]. The superscript notation on the time indicates the value just before $(-)$ or just after $(+)$ the kick.

To make the numerical calculation as efficient as possible, we use fast fourier transform techniques to perform the convolution in Eq. (26), but notice that in the exploration of small $\hbar$ values the dimension of the vector necessary to describe the entire wave function becomes excessively large. For this reason we limit the calculation to following the evolution of the ballistic peak, just as we did in the classical case. This procedure affords significant numerical advantages. Due to the finite bandwidth of the Floquet operator spectrum, only a small part of the wave function contributes to the peak dynamics. Thus, by concentrating our analysis on the peak, we are able to explore smaller values of $\hbar$ and/or longer times than has been done in previous calculations $[3,4,25]$. Nevertheless, according to the results obtained in Ref. [12], this procedure provides the same information concerning the anomalous dynamics that a complete wavefunction analysis would yield. This is certainly true for $\hbar$ so small and time so short as to make the correspondence principle valid. However, a deviation from the inverse powerlaw decay of the classical correlation function is a good indicator of the birth of quantum phenomena, even at very short times.

The calculational strategy of following the ballistic peak rather than the entire wave function is the most important aspect of our numerical treatment. This approach is based on the insight provided by the GOLD model where we saw that the time dependence of the ballistic peaks is given by the correlation function. An example of a complete calculation is shown in Fig. 4 where we reproduce one of the calculation of
Ref. [3] for a somewhat smaller value of $\hbar$. At first sight Fig. 4, like Fig. 2, may be interpreted as a strong confirmation of the analogy between the QKR and the CKR. In their calculation Hanson, Ott, and Antonsen [3] observed that the "sharp peaks" travel linearly in time and leave behind what they referred to as a "wake." From the solution to the GOLD model given by Eq. (8) we see that for sufficiently small values of Planck's constant that their wake is expected to eventually become a truncated Lévy distribution in momentum space and the peaks rather than being sharp have a finite area. Note, further, that the amplitude of the peaks is given by the correlation function of the dichotomous process and therefore this term ought to decay as an inverse power law. However, like Hanson, Ott, and Antonsen [3], we find that the intensity of the ballistic peak in Fig. 4 decays in time as an exponential, in striking contrast with the classical prediction (8). The reason for this discrepancy between the decay of the peaks in the QKR and the CKR is the fact that for large values of $\hbar$ the dominant mechanism for exiting the region of the accelerator mode is tunneling, something not available to the CKR.

Hanson, Ott, and Antonsen [3] find that a transition occurs, for the numerical value of the $\hbar$ used in the calculation smaller than a critical value, out of the region where the rate of exponential decay in time of the survival probability is $\exp [1 / \hbar]$ to some other dependence on $\hbar$, which is not determined analytically. Based on our analysis of the CKR and the GOLD model we expect an inverse power-law decay to be recovered as $\hbar \rightarrow 0$ in agreement with the correspondence principle. However, we find that the classical result is not fully recovered as $\hbar \rightarrow 0$ and effects of a purely quantum origin are present in all the explored $\hbar$ regions.

The initial condition for the QKR is a Gaussian wave packet to mimic the classical ensemble of initial conditions chosen in a way that the corresponding Wigner distribution has its maximum in proximity of the accelerator mode. Due to this particular choice of initial condition the Wigner function can be directly interpreted as a probability distribution that is then used to determine the initial conditions in the corresponding classical simulation. This allows for a direct comparison of the quantum and classical evolutions. Changing $\hbar$ obviously affects the width of the initial quantum distribution and therefore the set of classical initial states as well. In Fig. 3 we graph the area of the peak in the quantum calculations corresponding to different values of $\hbar$ as a function of time. We see that, for $\hbar$ smaller than $10^{-2}$, the area of the peak, $\Pi(t)$, exhibits the algebraic decay in time expected from the classical studies $[9,16]$. This type of decay implies that the dynamics of the QKR follows that of the CKR for small $\hbar$. On the other hand, for $\hbar>10^{-2}$, an exponential decay for the peak is found in agreement with that obtained in Ref. [3]. In the corresponding classical simulations no significant dependence on the initial distribution width has been detected.

The results obtained do appear, however, to depend in a subtle way on the location of the initial state. Further investigation of this sensitivity is required to completely understand the nonstationary nature of the phenomenon.

We find that in all the quantum calculation the inverse power-law behavior is eventually lost and replaced by an exponential decay. We interpret this second transition in 


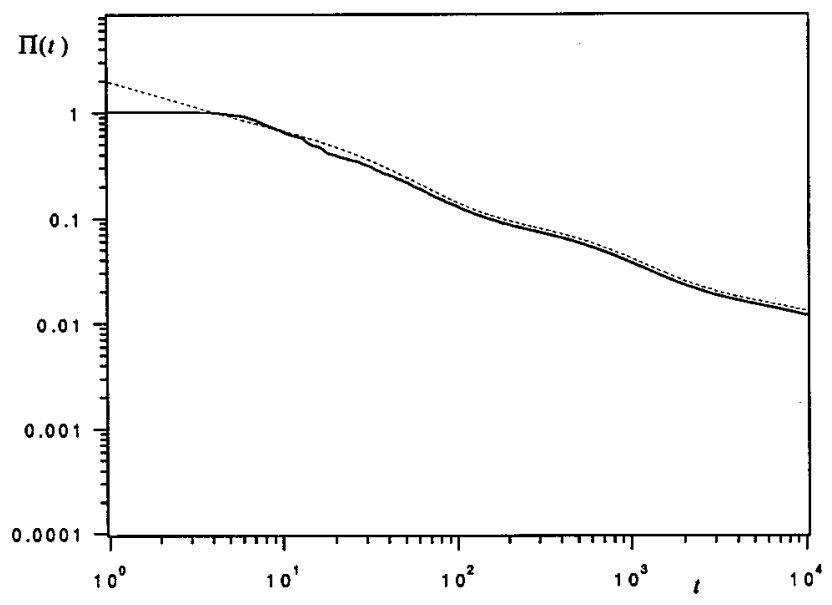

FIG. 5. Decay of the quantum peak for $\hbar=0.001$, solid line, and its numerical fit obtained by using Eq. (33). The fit is shifted for graphical purposes. The normalization on $\Pi(t)$ is $\Pi(1)=1$.

terms of two phenomena, namely, the tunneling between the fractal region of the cantori and the stochastic sea, and the quantum induced breakdown of classical self-similarity [4]. We do not pursue an investigation into this effect here, but rather leave it as a subject for future work. Thus, leaving the eventual crossover from inverse power law to exponential decay aside, we see that the QKR and CKR behave similarly, both having an inverse power-law decay for sufficiently small $\hbar$. However, the decay of the peak intensity in the quantum calculation is modulated, with the modulation function being periodic in the logarithm of the time. The logarithmic oscillations suggest a renormalization group approach to an understanding of the quantum decay.

\section{LOGARITHMIC OSCILLATIONS AS A MANIFESTATION OF QUANTUM COHERENCE}

We have seen that the numerical calculations of the QKR show the ballistic peaks decaying as modulated inverse power laws in time, eventually going over to an exponential decay. This picture is made compelling by the extremely good agreement between the numerical results and the fitting function

$$
\Pi(t)=\frac{A_{1}+A_{2} \cos (Y \ln t)}{t^{\beta}} \exp (-\lambda t)
$$

as shown in Fig. 5. The function (27) has five parameters $A_{1}, A_{2}, \beta, \lambda$, and $Y$ that are fit to the results of the numerical calculation using a mean-square minimization program Equation (27) has the form of the solution to the renormalization equation in Sec. III, Eq. (12), when $\lambda=0$. However, in spite of the fact that the scaling relation produces logarithmic oscillations we shall see that the frequencies of the oscillations depicted in Fig. 5 do not behave as those in Eq. (13). We see from the general solution to (12) given in [26] that upon increasing the value of $\ln b$, the period of the logarithmic oscillations, the intensity of the oscillations increases. This behavior is not consistent with the correspondence principle, which, since the oscillations are not present in the CKR calculations, would suggest that the intensity of the oscillation for the QKR should disappear as $\hbar \rightarrow 0$, not

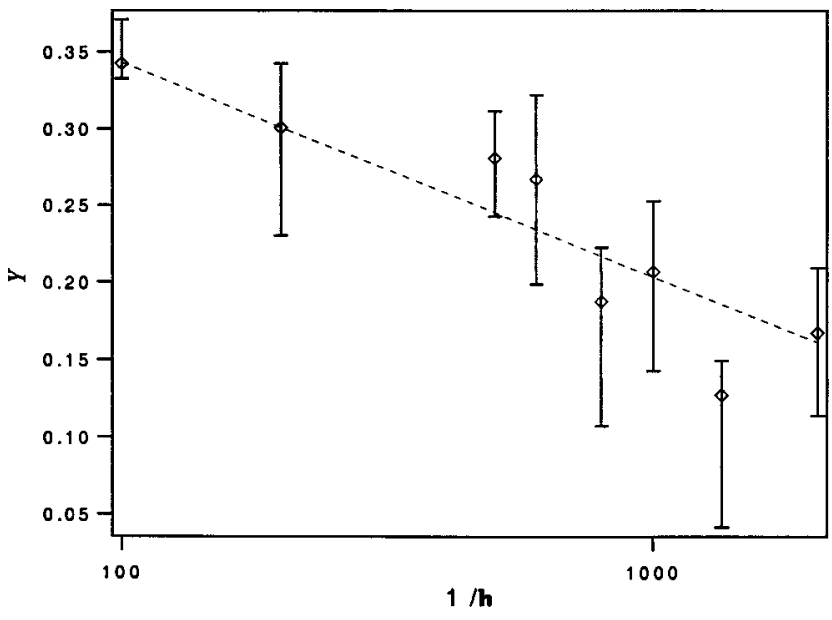

FIG. 6. The dependence of the renormalization group oscillation dimensionless frequency $Y$ in Eq. (33) on $1 / \hbar$ as obtained by fitting the quantum peak decays. The dashed line denotes a power-law fitting with slope $\alpha=0.14$

increase. Thus, as the frequency decreases (period increases) the intensity of the oscillations should go to zero. This latter behavior is observed in Fig. 6 where the frequency of the oscillation in the QKR is plotted versus $\ln (1 / \hbar)$. Therefore the source of the quantum modulation requires the introduction of a new physical mechanism.

We are guided by the correspondence principle and the renormalization group properties we wish to retain in our selection of the quantum form of $\phi(t)$ to replace Eq. (11). The exact correspondence between the classical and quantum calculations is lost on a time scale given by Berry [27] as $t_{B} \propto(1 / \gamma) \ln (1 / \hbar)$ with $\gamma$ being the Lyapunov exponent for the process. After this time there is an extended interval during which the dominant behavior of both calculations of survival probability is the inverse power-law decay, resulting from the scaling properties of both the CKR and QKR. We assume a quantum mechanism to account for the existence of oscillations in the QKR that has no classical analogue. This mechanism accounts for the transitions between accelerator modes that works in concert with the classical mechanism and which retains coherence across the fractal boundaries. We notice that a straightforward way of modifying Eq. (13), to incorporate this coherence effect, is to assign a phase to the quantum transition rate;

$$
\tilde{w}=w e^{i B},
$$

so that the scaling relation found earlier is replaced with

$$
\tilde{\phi}(\tilde{w} t) \approx \frac{1}{\left(\tilde{w}^{2} q\right)} \tilde{\phi}(t)
$$

and the quantum coherent effect is given by the real part of the scaling function. The solution to Eq. (29) is of the same form as that given earlier, a modulated inverse power law, except that the inverse power-law index is complex and may be thought of as a complex fractal dimension [28]:

$$
\alpha=2+\frac{\ln q}{\ln q+i B}=\mu-i \kappa,
$$

where the real part of the index is 


$$
\mu=2+\frac{\ln q \ln w}{(\ln w)^{2}+B^{2}}
$$

and the imaginary part of the index is

$$
\kappa=\frac{B \ln q}{(\ln w)^{2}+B^{2}}
$$

We therefore obtain

$$
\operatorname{Re} \tilde{\phi}(t) \approx \frac{A_{2} \cos (\kappa \ln t)}{t^{\mu}}
$$

and when the "coherence strength" is very small, $B / \ln w$ $\ll 1$, this reduces to

$$
\operatorname{Re} \tilde{\phi}(t) \approx \frac{A_{2} \cos (Y \ln t)}{t^{\mu}}
$$

where

$$
Y \equiv \frac{B}{\ln w}(\mu-2)
$$

To recover the form of the fitting function we recall that the frequency of oscillation (35), must vanish as $\hbar \rightarrow 0$, so that Eq. (34) cannot be the sole contribution to the quantum waiting time distribution function. Therefore to obtain the proper scaling behavior of the waiting time distribution in the classical limit we add the contribution of (34) and (13) to obtain

$$
\phi_{\text {quan }}(t) \approx \frac{A_{1}}{t^{\mu}}+\operatorname{Re}\left[\frac{A_{2}}{t^{\alpha}}\right]=\frac{A_{1}+A_{2} \cos \left(\frac{2 \pi \ln t}{\ln T}\right)}{t^{\mu}} .
$$

Here the complex fractal dimension is given by $\alpha=\mu$ $+i 2 \pi / \ln T$, and the imaginary part of the fractal dimension gives the period of modulation of the inverse power law, see Ref. [28], and $\ln T=2 \pi \ln w /[B(\mu-2)]$ is the period of oscillation induced by the coherence mechanism.

We have seen that the inverse-power-law structure modulated by logarithmic oscillations remains invariant upon differentiation and integration so the structure of the correlation function and the area of the peak will maintain the same structure. In the formula we use to fit the decay of the area of the peak (27) we include also a long-time exponential decay that does not have any justification in the present model, but was detected in the calculations and may play an important role in future work. The power-law index $\beta$ in Eq. (27) is expected to be the same as in the classical case

$$
\phi(t)=\lim _{\hbar \rightarrow 0} \phi_{\text {quan }}(t)
$$

since in the case of very small Planck's constant, the time after which the exponential decay becomes important lies beyond the range of our observation, i.e., the parameter $\lambda$ is always extremely small. Further, to ensure that $\Pi(t)$ is positive definite we must have that $A_{1}>A_{2}$. We focus on the adoption of the fitting formula (27) to determine the parameter $\ln T$ and from it the coherence phase $B$, which must satisfy

$$
\lim _{\hbar \rightarrow 0} B=0
$$

The determination of this crucial parameter allows us to assess the amount of quantum coherence present in the seemingly classical behavior. In Fig. 6 we show the dependence of the frequency of oscillation of the waiting time distribution function on the value of Planck's constant used in the calculation. From the negative slope of the curve we reach the conclusion that the coherence parameter $B$ must have the functional form

$$
B \propto \hbar^{\alpha}
$$

with $\alpha \simeq 0.14$. Thus, the coherence resulting in logarithmic oscillations is a genuinely quantum effect, due to the dependence of the frequency of oscillation on $\hbar$, and seems to be a slowly decaying function of $\hbar$ so as to vanish in the classical limit.

\section{CONCLUSIONS}

We see from Fig. 5 that the agreement between the fitting function and the numerical results are so good as to make it impossible to distinguish the numerical result from the fit. We think that this is compelling evidence that the logarithmic oscillations are not an artifact of the calculational technique. Furthermore, due to the fact that the classical case does not contain such oscillations for the parameter values chosen in the calculation, but results an unmodulated inverse power-law behavior, we reach the conclusion that these oscillations are of a quantum origin.

We distinguish a number of different time domains in the present calculation. First of all is the time scale $t_{B}$ identified by Berry [27] and such that the results of the classical and quantum cases diverge where $t_{B} \propto(1 / \gamma) \ln (1 / \hbar)$ with $\gamma$ being the Lyapunov exponent for the process. This time scale is fairly short and is observed in Fig. 3, where we see the onset of logarithmic oscillations in under 10 kicks that are observed quantum mechanically, but not classically. This is also the time scale on which Hanson, Ott, and Antonsen [3] account for the exponential decay of the survival probability by means of tunneling. The second time scale occurs after this tunneling region for values of Planck's constant, $\hbar$ $\leqslant 10^{-2}$, after which time the mechanism for escape is no longer tunneling so the decay transitions from exponential to inverse power law. Thus, after the crossover, anomalous diffusion appears in the quantum domain [4]. Even if the quantum and classical results diverges at early time (for $t<100$ ) [4], the subsequent quantum behavior resembles the classical one, namely, that the area under the peak shows an inverse power-law decay, however, one that is modulated with logarithmic oscillations. This behavior is eventually dominated by exponential decay, which is not necessarily related to the tunneling processes found by Hanson, Ott, and Antonsen [3], but rather may be related to the reemergence of the wave function in the chaotic sea for small values of Planck's con- 
stant. We shall study this late-time breakdown in detail in a forthcoming paper.

The quantum intermediate time region, where the correspondence between the classical and quantum evolution is lost, but retains the overall power-law decay, has been carefully explored in this paper. We found that quantum mechanical properties show up under the form of oscillations around the expected inverse power-law decay of the peaks. We have established that these oscillations can be reproduced very well adopting a fitting function the structure of which is defined on the bases of renormalization group arguments, including a genuine quantum coherence mechanism.

Furthermore, we have to say that only a few oscillations can be detected. At very small values of $\hbar$ the frequency

[1] B. V. Chirikov, Phys. Rep. 52, 265 (1979).

[2] L. Reichl, The Transition to Chaos (Springer-Verlag, New York, 1992).

[3] J. D. Hanson, E. Ott, and T. M. Antonsen, Phys. Rev. A 29, 819 (1984).

[4] R. Roncaglia, L. Bonci, B. J. West, and P. Grigolini, Phys. Rev. E 51, 5524 (1995).

[5] D. L. Shepelyanshy, Physica D 8, 208 (1983); G. Casati et al., Phys. Rev. Lett. 56, 2437 (1986).

[6] F. M. Izrailev, Phys. Rep. 196, 229 (1990).

[7] E. W. Montroll and B. J. West, in Fluctuation Phenomena, 2nd ed., edited by E. W. Montroll and J. L. Lebowitz, Studies in Statistical Mechanics (North-Holland, Amsterdam, 1987), Vol. 7.

[8] E. W. Montroll and M. F. Shlesinger, in From Stochastics to Hydrodynamics, edited by J. L. Lebowitz and E. W. Montroll (North-Holland, Amsterdam, 1984).

[9] G. Zumofen and J. Klafter, Phys. Rev. E 47, 851 (1993).

[10] M. F. Shlesinger and G. Zaslavsky, Editors, Lévy Flights and Related Topics in Physics (Springer, Berlin, 1994).

[11] H. E. Stanley, Introduction to Phase Transitions and Critical Phenomena (Oxford Science, Oxford, 1971).

[12] P. Allegrini, P. Grigolini, and B. J. West, Phys. Rev. E 54, 4760 (1996).

[13] J. Klafter, G. Zumofen, and A. Blumen, Chem. Phys. 177, 821 (1993); J. Klafter and G. Zumofen, Phys. Rev. E 49, 4873 (1994); J. Klafter, G. Zumofen and M. F. Shlesinger, in ChaosThe Interplay Between Stochastic and Deterministic Behaviour, Proceedings, Karpacz, Poland 1995, edited by P. Garbac- becomes extremely low making it very difficult to see more than two or three oscillations while with bigger values of $\hbar$ an early transition to normal diffusion and so to exponential decay of the peak cancels the effect [3]. In conclusion, the meaning of the numerical results of this paper is that deviations from the classical behavior occurs from the earliest evolution under the form of a quantum generated modulation of the classical results.

\section{ACKNOWLEDGMENTS}

We thank the Italian CNR and NATO, as well as the Office of Naval Research, for partial support of this work. zewski, M. Wolf, and A. Weron (Springer, Berlin, 1995), p. 183.

[14] B. J. West, P. Grigolini, R. Metzler, and T. F. Nonnenmacher, Phys. Rev. E 55, 99 (1997).

[15] T. Geisel, J. Nierwetberg, and A. Zacherl, Phys. Rev. Lett. 54, 616 (1985); T. Geisel, A. Zacherl, and G. Radons, Z. Phys. B 71, 117 (1988).

[16] G. Zumofen and J. Klafter, Europhys. Lett. 25, 565 (1994).

[17] J. Klafter, G. Zumofen, and M. F. Shlesinger, in Lévy Flights and Related Topics in Physics (Springer, Berlin, 1994).

[18] M. Stefancich, P. Allegrini, L. Bonci, P. Grigolini, and B. J. West (unpublished).

[19] R. Ishizaki, T. Horita, T. Kobayashi, and H. Mori, Prog. Theor. Phys. 85, 1013 (1991); R. Ishizaki, H. Hata, T. Horita, and H. Mori, ibid. 84, 179 (1990).

[20] J. D. Hanson, J. R. Cray, and J. D. Meiss, J. Stat. Phys. 39, 27 (1985).

[21] B. J. West and W. Deering, Phys. Rep. 246, 1 (1994).

[22] R. Bettin, R. Mannella, B. J. West, and P. Grigolini, Phys. Rev. E 51, 212 (1995).

[23] E. Floriani, R. Mannella, and P. Grigolini, Phys. Rev. E 52, 5910 (1995).

[24] B. Eckhardt, Phys. Rep. 163, 205 (1988).

[25] L. Bonci, P. Grigolini, A. Laux, and R. Roncaglia, Phys. Rev. A 54, 112 (1996).

[26] B. J. West, J. Opt. Soc. Am. A 7, 1074 (1990).

[27] M. Berry, Proc. R. Soc. London, Ser. A 400, 229 (1985).

[28] M. F. Shlesinger and B. J. West, Phys. Rev. Lett. 67, 2106 (1991). 